\title{
The circadian clock and hypoxia in tumor cell de-differentiation and metastasis
}

\author{
Lasse Jensen
}

\section{Linköping University Post Print}

\section{Tweet}

N.B.: When citing this work, cite the original article.

Original Publication:

Lasse Jensen, The circadian clock and hypoxia in tumor cell de-differentiation and metastasis, 2015, Biochimica et Biophysica Acta - General Subjects, (1850), 8, 1633-1641.

http://dx.doi.org/10.1016/j.bbagen.2014.10.025

Copyright: Elsevier

http://www.elsevier.com/

Postprint available at: Linköping University Electronic Press

http://urn.kb.se/resolve?urn=urn:nbn:se:liu:diva-120029 


\title{
The circadian clock and hypoxia in tumor cell de-differentiation
}

\section{and metastasis}

\author{
Lasse Dahl Jensen ${ }^{1,2 *}$
}

${ }^{1}$ Department of Microbiology Tumor and Cell Biology, Karolinska Institute, 17177

Stockholm, Sweden

${ }^{2}$ Department of Medical and Health Sciences, Linköpings University, 58185 Linköping

*Correspondence should be addressed to Dr. Lasse Jensen, lasse.jensen@liu.se

Keywords: Circadian, clock, hypoxia, zebrafish, vasculature, endothelium, disease models, differentiation, de-differentiation, angiogenesis, reactive oxygen species, nitric oxide, cancer, metastasis, EMT. 


\begin{abstract}
BACKGROUND: Cancer is considered to develop due to disruptions in the tissue microenvironment in addition to genetic disruptions in the tumor cells themselves. The two most important microenvironmental disruptions in cancer are arguably tissue hypoxia and disrupted circadian rhythmicity. Endothelial cells, which line the luminal side of all blood vessels transport oxygen or endocrine circadian regulators to the tissue and are therefore of key importance for circadian disruption and hypoxia in tumors. SCOPE OF REVIEW: Here I review recent findings on the role of circadian rhythms and hypoxia in cancer and metastasis, with particular emphasis on how these pathways link tumor metastasis to pathological functions of blood vessels. The involvement of disrupted cell metabolism and redox homeostasis in this context and the use of novel zebrafish models for such studies will be discussed. MAJOR CONCLUSIONS: Circadian rhythms and hypoxia are involved in tumor metastasis on all levels from pathological deregulation of the cell to the tissue and the whole organism. Pathological tumor blood vessels cause hypoxia and disruption in circadian rhythmicity which in turn drives tumor metastasis. Zebrafish models may be used to increase our understanding of the mechanisms behind hypoxia and circadian regulation of metastasis. GENERAL SIGNIFICANCE: Disrupted blood flow in tumors is currently seen as a therapeutic goal in cancer treatment, but may drive invasion and metastasis via pathological hypoxia and circadian clock signaling. Understanding the molecular details behind such regulation is important to optimize treatment for patients with solid tumors in the future.
\end{abstract}




\section{Introduction}

The ability to adapt to predictable changes in the environment is of major importance in physiology. The most predictable and recurring environmental variable is arguably that of the daily change in light and darkness. As these oscillations occur with a constant period of close to 24-h, they form the basis of what is known as circadian (circa: approximately, diem: a day) rhythms in all living organisms. In most organisms, many aspects of physiological processes including activity/rest cycles, metabolism, proliferation, biological defense systems and regulation of cellular specification are tightly coupled to the molecular circadian clock which relay the light/dark information on both cellular, tissue and organismal levels[1]. The development of more complex cellular phenotypes and functions in higher organisms has happened under the influence of the circadian clock. As a consequence, the circadian clock has come to play a major role in the regulation of differentiation as well as pathological dedifferentiation in for example cancer, often via crosstalk with other pathways involved in this processes $[2,3]$. Among these other pathways, signaling through oxygen-sensors is one of the most important co-regulators of cellular differentiation (reviewed in [4]).

Oxygen is one of the most vital, and at the same time most damaging natural compounds. Thus, all cells have developed specialized systems to utilize and protect against oxygen - or the lack thereof, a condition known as hypoxia. Hypoxia and circadian regulation are furthermore tightly coupled as oxygen use and management of oxygen bi-products (reactive oxygen species, ROS) exhibit circadian variation due to changes in activity and metabolism 
during the day vs. the night (reviewed by [5]). In mammals, the embryo experience cycles in hypoxia and normoxia during development coupled to distinct phases of vascular development and remodeling in various tissues[6, 7]. Hypoxia and hypoxia-induced reactive oxygen species are therefore among the most important physiological regulators of cellular differentiation/de-differentiation. During progression of diseases such as cancer, these pathways become re-activated leading to pathological de-differentiation and re-acquisition of a motile phenotype by invasive tumor cells, a change that is brought about by tissue hypoxia in the expanding tumor mass[4]. Tumor hypoxia may therefore be one of the most important pathogenic factors in cancer, indicating that a tumor has progressed to an invasive lesion and thus implying a poorer prognosis for the patient[8].

Circadian rhythm- and hypoxia-signaling are currently subject to rigorous mechanistic research aimed at elucidating molecular regulators of these pathways in cells. However, a lack of disease models in which these pathways can be studied under controlled conditions have delayed the progress in our understanding of how hypoxia and the circadian clock contribute to cancer and in particular pathological de-differentiation and metastasis. Zebrafish have in recent years emerged as a powerful model to study hypoxia and circadian rhythms in a pathologically relevant manner during disease processes that accurately recapitulate those seen in human cancer and in particular during tumor metastasis[9-11]. Zebrafish embryos, and some adult strains are transparent, and develop outside the womb, meaning that light reaches and entrains all cells. As such, molecular or physiological responses to normal or disrupted light/dark cycles can be studied directly without interference from the circadian clock of the mother. The zebrafish embryo is also optimal for studies of tissue hypoxia. During the first week of development, the embryo is so small that all cells receive sufficient oxygen by passive diffusion from the water, rendering their oxygenation state unaffected by circulation and instead directly related to the amount of oxygen in the aqueous medium[12, 
13]. As responses to radical changes in oxygenation cannot be studied as an isolated event without also compromising perfusion in any other vertebrate model organism, this makes zebrafish highly attractive for physiologic or mechanistic studies on the effects of hypoxia in vivo. We have recently developed highly robust methods to dynamically and accurately manipulate tissue oxygenation during zebrafish development $[14,15]$. These models give full control over the amount and duration of tissue hypoxia and the ability to switch between any degree of tissue oxygenation at will. The zebrafish system is therefore well suited for studies on hypoxia and circadian regulation of cellular differentiation states, and has been used to generate important information on how this is regulated in cancer.

In this review, I will discuss the molecular basis for circadian and hypoxia signaling in cells and on the level of the whole organism. I will focus on what is known about their involvement in regulation of cellular differentiation/de-differentiation with a particular focus on their crosstalk with cellular redox-signaling pathways in cancer metastasis. I will throughout the review highlight contributions from zebrafish models.

\section{The circadian clock and cancer}

The circadian clock is organized almost identically in all cells including light-sensitive retinal ganglion cells, food-sensitive hepatocytes, skin fibroblasts or any other cell type - at least at its core (Figure 1). Entraining cues, such as activators of retinoic acid orphan nuclear receptor (ROR)alpha activity, lead to activation of ROR-elements (RORE) and transcription of the bmall gene (also known as arntl) during the day in diurnal organisms (reviewed in [16]). Bmal1 proteins heterodimerize with Clock or Npas2 and as such constitute the DNAbinding portion of the circadian transcriptional activator. This complex drives transcription of circadian output genes (CCGs) including Period1-3, Cryptochrome1-2 and Rev-Erb-alpha. 
These factors in turn act as transcriptional repressors inhibiting both their own transcription as well as that of other CCGs (Figure 1). This core negative feedback loop is sufficient to maintain clock oscillations, but insufficient for maintaining robust 24-h rhythms. Casein kinases have been shown to elicit phosphorylation of the Bmal1, Period and Cryptochrome proteins, which regulate their nuclear entry and transcriptional activities[17, 18]. Bmal1, Periods and Cryptochromes are furthermore degraded by various ubiquitin ligases $[19,20]$ and as such their abundance in the cell is differentially regulated, which is important to confer the 24-h period length on circadian outputs both in vitro and in vivo.

In contrast to the core circadian clock, the circadian outputs may differ between cell types. Some tissues specialize in circadian secretion of endocrine factors such as melatonin produced in the pineal gland, cortisol produced in the adrenal cortex and insulin produced in the pancreatic islets, which keep the clock running smoothly in other tissues (reviewed in [21]). The core clock is, however, important for cell physiology in general, as many metabolic and house-keeping processes are under circadian control. Therefore specific clock disruption only in particular cells types such as endothelial cells, smooth muscle cells, adipocytes, muscle cells, neurons or macrophages, to mention a few, always seem to disrupt the function of that particular cell type [22-25], which in almost all cases lead or contribute to pathologies, even when the global circadian rhythm in the rest of the organism is undisturbed. It is therefore not surprising that cancer cells often are found to harbor mutations in clock, or clock-associated factors, which enable pathological changes in the cells important for establishing a malignant lesion.

2.1 Role of circadian clocks in cancer 
Genetic alterations in the core clock proteins are frequently found in a variety of cancers (reviewed by [26]). However, even in cases where such mutations are lacking, tumor cells may still exhibit disrupted circadian signaling. Entraining cues such as glucose, cortisol, melatonin or insulin will not exhibit clear rhythmic changes in their local concentration in the tumor at advanced stages due to chaotic and ineffective perfusion through the tumor blood vessels (more details on the nature of tumor blood vessels later in this review). As such, circadian oscillation in the serum levels of endocrine circadian mediators may not be sensed by the tumor as the blood is retained within or not entering into the tumor as in healthy tissues. Also, the receptors eliciting induction of the circadian clock in response to these factors, such as the melatonin receptor (MT)1 have been found to be mutated and dysfunctional in tumors of some patients, which significantly correlated with reduced survival [27]. Finally the CCGs are frequently mutated in tumor cells either in their coding sequence and/or the regulatory elements in which the circadian factors bind (the E-boxes) leading to a failure in the circadian regulation of physiologic responses in which they are involved[26]. The overall consequence is that tumor cells within the tumor have lost their circadian behavior, and this is an important transformation which enables other essential pathologic features of cancer. For example, we have recently found that the circadian clock is a strong regulator of the VEGF promoter, leading to drastic differences in VEGF production during the day and the night in developing zebrafish embryos[10, 28]. This regulation was brought about by Bmal1 binding to E-boxes present in the promoter region of VEGF, which is crucial for activating transcription; without Bmal1 or if these E-boxes are deleted from the promoter, transcription of the vegf gene was effectively silenced. We further found that Period 2 is a strong repressor of vegf transcription during the day. Knock-down of Period2 led to augmented VEGF-production, especially in light-at-night conditions in which Period2 levels are elevated and VEGF-levels as well as developmental angiogenesis is reduced[28]. A 
similar mechanism may be in play in mice, as mothers kept in constant darkness during their pregnancy gave birth to pubs which exhibited elevated VEGF-A levels, coupled to impaired regression of hyaloid vessels and retinal vascular overgrowth during the first week after birth[29]. While these findings proved that VEGF is regulated by the circadian clock during development in zebrafish and mice, other reports suggest that a similar mechanism may be in place in adult cells as well, and that such regulation is disrupted in cancer. As such, Period2 and Cryptochrome1 have been found to inhibit VEGF-production in tumor cells, especially in response to hypoxia[30]. Period2 has been proposed to be a tumor-suppressor as mice lacking period 2 are sensitized to develop teratomas following irradiation[31], and tumor cells which have been transduced to express constitutively high levels of Period2 grow slower in xenograft-models[32]. These findings indicate the loss of period 2 in tumor cells and thus circadian clock dysfunction could play an important role in pathological hypoxia-induced VEGF hyper-expression, leading to accelerated tumor angiogenesis and a more invasive phenotype of the tumor. Also in humans, this mechanism seems to be conserved. Bmal1, Npas2 and Clock have been indicated as important for VEGF-production during the night[33, 34]. In agreement with these findings, Clock is often up-regulated and Period 2 downregulated in human colorectal cancer and the levels of tumor Clock expression exhibit a tight positive correlation with tumor VEGF-levels and metastatic dissemination in patients[35, 36]. Collectively these findings illustrate the importance of the circadian clock for regulation of tumor angiogenesis and metastasis.

Importantly, circadian factors have also been implicated in maintaining progenitor cell health and as such to hinder premature senescence. As such, Period2 is important for healthy functions and recruitment of endothelial progenitor cells to growing vessels in mice; animals in which the period 2 gene has been mutated and rendered dysfunctional exhibit vascular senescence and a lack of ischemia-induced angiogenesis in the hind-limb muscle 
tissue [37]. In addition to the direct regulation of tumor cell dedifferentiation by the pathological tumor cell circadian clock[3], clock disruption also lead to elevated intracellular ROS[38], which in turn could drive de-differentiation. In fact, the circadian clock has been extensively implicated in redox-biology. In mice with a de-regulated clock, such as in Bmal1$\mathrm{KO}$ or Clock-mutant mice, endothelial nitric oxide synthase (eNOS) activity is uncoupled producing large amounts of superoxide instead of $\mathrm{NO}$ [38]. Furthermore, redox enzymes such as peroxiredoxins have been shown to exhibit robust circadian rhythms implying that redox homeostasis is under control of the circadian clock machinery in cells [39].

In tumors, innervation or direct light-stimulation is probably insufficient for effective entrainment of the circadian clock. Thus, entrainment relies on signaling by endocrine circadian mediators. Such mediators, which include melatonin, cortisol, insulin, glucose, free fatty acids and various hormones and growth factors are all delivered via the circulation, and therefore the vasculature play a key role in entrainment of tumor tissues. The tumor vasculature, however, is immature as it is constantly exposed to angiogenic stimuli [40, 41]. Tumors have as such been characterized as a "wound that do not heal" in which the vasculature is highly tortuous, exhibit large vascular plexuses and aneurisms, lack mural cell support and proper endothelial cell-cell contacts rendering the vessel wall unstable and highly leaky[42, 43]. Furthermore, perfusion is often inhibited due to high interstitial fluid pressure (brought about by the leaky nature of the vessels as well as mechanical tension within the tumor) and a failure to induce specification of arterial or venous vascular phenotypes leading to blood being transported in both directions (afferent and efferent) in the same vessel. Many vessels are also collapsed. Collectively these pathological vascular phenotypes lead to intratumoral hypoxia even in highly vascularized tumors[44]. In addition, tumor cells may incorporate into the vessel wall and thus constitute part of the endothelium - a process known as vasculogenic mimicry[45], which may be a route for preserving a minimum of circulation 
even after therapy-induced vascular regression. The consequence of all these pathological features of tumor blood vessels is that blood will not pass through the tumor at the same rate as healthy tissues, thus reducing the functionality of the tumor vasculature (reviewed further in [46]). As such, the rhythm of blood-borne signaling factors may not be circadian, at least not in all parts of the tumors. This would affect the circadian rhythm of the tumor cells, but also of the endothelial cells in the tumor vasculature, which could play a role in vesselinitiated metastasis. As such, it is known that genetic disruption of the endothelial clock lead to vascular senescence, elevated ROS levels, impaired NO production and impaired maintenance of the vascular basement membrane leading to vascular stiffness and pathological arterial remodeling in hypertensive arteries[37, 38, 47]. These pathological changes in the endothelial cells could potentially lead to sustained inflammatory activation and unproductive angiogenesis in the tumor[48], reduced vessel repair after stress injury, reduced vascular stability and barrier function[46] as well as further disruptions of tumor perfusion and hypoxia - all of which are important in tumor cell de-differentiation and metastasis. In the future it will be important to directly investigate whether circadian disruption in endothelial cells would be directly involved in tumor dissemination and metastasis.

\section{Hypoxia signaling in cancer}

Oxygen is a key regulator in mammalian metabolism due to its use as the terminal electron acceptor in cellular energy production in the mitochondria. Therefore, should oxygen be lacking in the environment, the cell need to respond quickly to adapt its physiology accordingly. As such, the most well-studied hypoxia-response pathway has developed such that the crucial signaling factor, HIF-1alpha, is continuously produced but also degraded in 
normoxia via oxygen-induced hydroxylation by members of the prolyl-dehydroxylase (PHD)s family which target the protein for recognition and ubiquitinylation by the von hippel-lindau (VHL) E3 ubuiquitin ligase complex and subsequent degradation by the proteasome (reviewed in [49]) (Figure 2). Under hypoxia, however, PHDs are inactive leading to stabilization of pre-produced HIF-1alpha, which become free to dimerize with HIF-1beta (also known as ARNT) and activate transcription of target genes through binding to hypoxia-responsive elements (HRE) in the promoter region[49]. Among such targets are genes involved in regulation of tissue perfusion (ie eNOS and VEGF) and production of erythrocytes (ie Erythropoietin), the function of which are to increase oxygenation of the hypoxic tissue by increased perfusion and oxygen binding capacity of the blood[50, 51]. The cells also adapt by reducing the amount of oxygen consumed through a switch to glycolysis rather than oxidative metabolism, which require more glucose uptake and therefore an upregulation of glucose transporters (especially Glut4), while at the same time reducing energy demanding processes such as cell division[52]. HIFs may also be regulated by other factors such as factor inhibiting hif (FIH), or by sumoylation (reviewed further in [49]), which reduce the transcriptional activity or provide a protection against ubiquitinylation respectively. Also non-HIF pathways have been reported to be important for hypoxia signaling. For example, ER-stress resulting from hypoxia-induced inhibition of proper disulphide bond formation leads to activation of the unfolded protein response (UPR). Of particular importance in cancer, hypoxia and reoxygenation-induced ROS formation induce DNA double strand brakes thus activating ataxia telangiectesia mutated (ATM) signaling [53]. Thus the response to hypoxia is complex and cannot be accurately recapitulated by modulation of the HIF-1, or any other single hypoxia-induced pathway, alone - in particular not in cancer. 
Zebrafish are remarkably tolerant to hypoxia, much more so than air-breathing animals, and therefore constitute a valuable system for studies in this field. Zebrafish embryos adapt their metabolism to the amount of oxygen present, and therefore enter into a state of suspended animation rather than die when experiencing extremely low concentrations of oxygen in their surroundings[54]. Adult zebrafish are also impressively hypoxia-tolerant and can easily stand as little as $10 \%$ of the oxygen in fully air-saturated water for extended periods of time[11, 55]. Our and other laboratories have in order to take advantage of these features, developed systems particularly adapted for manipulation of oxygen levels in the water. As the zebrafish embryo in particular exhibit extremely high oxygen consumption, oxygen concentration gradients are expected to develop around the embryo if the water is stagnant. In our system we therefore secure constant flow of water over the embryos by uninterrupted, gentle stirring $[14,15,50]$. The water oxygen levels are adjusted by perfusion of nitrogen gas into the water, which is controlled by coupling the gas-flow to a valve that shut off when a particular user-determined oxygen tension has been reached, measured by an inline oxygen electrode. The system is practically identical for adult zebrafish (Figure 3), but the aquarium is usually larger in order to accommodate more fish at the same time. For studies on the effects of acutely changing oxygen levels in the fish, we have developed a system in which the fish can be taken from a holding aquarium in which it has been adapted to one level of oxygen (usually normal, fully oxygenated water) and be placed in a tube perfused with pre-equilibrated water of a different oxygen tension (usually hypoxic water)[11]. While in the tube, the fish can be monitored and analyzed and as such, dynamic events such as the acute hypoxia-induced increase of blood flow through otherwise nonperfused (lymphatic-like) vascular beds have been observed.

In our systems, we utilize a semi-sealed aquarium in which oxygen-rich air slowly leak in causing a slow increase in water oxygen tension until the level is above a hysteresis- 
threshold which will then trigger the re-engagement of the nitrogen perfusion, lowering the oxygen tension back to its set level[ $[15,55]$. Others have instead utilized fully sealed systems in which the water oxygen tension is initially prepared in a similar way but then the nitrogen gas is switched to a source of oxygen, which is slowly applied to the water in order to compensate for the oxygen-consumption of the fish[56]. This may at least hypothetically lead to a buildup of $\mathrm{CO}_{2}$ /bicarbonate in the water, which affect the $\mathrm{pH}$ and possibly cause hypercapnia in the fish. Such problems were however not reported even after several weeks in such aquaria, which may have been due to the large water volumes used. Also in other setups, systems to study hypoxia in fish have focused on regulating water-oxygen levels through controlled addition of gasses to the water itself [57], rather than to the air-phase above the water, which is the most common way to expose cells (which also live in aqueous medium) to hypoxia.

In addition to environmental hypoxia models zebrafish strains have been generated in which key factors involved in the signaling pathways responsible for mounting the cellular responses to hypoxia were mutated and rendered non-functional[58, 59]. In one such genetic hypoxia models, the VHL protein was inactivated thus creating a "chronically hypoxic" zebrafish strain in which HIF1-signaling is always ongoing, in spite of de facto proper oxygenation of all the cells in the fish[59]. These embryos exhibit many classic hypoxic phenotypes including hyperventilation, hypervascularization and increased erythropoiesis[59]. While HIF-1 is very important in hypoxia signaling, there are also other hypoxia pathways, which may not be de-regulated while also VHL may be involved in other signaling pathways in addition to hypoxia-signaling, in this genetic mutant. Caution should, therefore, be taken when using this or other mutant zebrafish strains as a hypoxia-readout. Hypoxia-signaling can also be induced chemically[60]. Most such approaches focus on inhibition of PHD-induced hydroxylation of HIF-1, upstream of VHL by drugs such as 
phenylhydrazine [60], DMOG[59] or $\mathrm{CoCl}_{2}[11]$. However as most drugs are not exclusively targeting one protein, these methods seem even more inaccurate as ways to gage effects of hypoxia. I therefore recommend that pathologically relevant, true hypoxia models such as the ones described in Figure 3 are always used instead of or in combination with genetic or pharmacologic "pseudo-hypoxia" models.

\subsection{Role of hypoxia in EMT and metastasis}

Hypoxia is a key factor driving pathological changes in the tumor microenvironment, which are necessary for enabling tumor development, growth, invasion, metastasis and resistance to treatment[4]. During early tumorigenesis, hypoxia results from the physical expansion of the hyperplastic cell mass coupled with high metabolic activity of these cells. Thus, some of the tumor cells will eventually be localized too far away from the existing vasculature for efficient oxygenation and glucose delivery, enabling hypoxia-signaling in these cells. Such cells respond by producing VEGF and other angiogenic molecules, which induce tumor angiogenesis, a crucial element for progression of hyperplastic lesions past premalignant or dormant stages[61]. As the tumor grows larger, hypoxia also affect tumor stromal cells including endothelial cells, tumor associated macrophages (TAMs), cancer associated fibroblasts (CAFs) and perivascular mural cells such as pericytes and smooth muscle cells $[4,9,50]$. In response to hypoxia, these cells aid in tumor progression and resistance to therapy by producing growth and survival factors as well as by opening up the vasculature and ensuring systemic dissemination and metastasis[62, 63]. For example, it was recently discovered that tumor hypoxia drives the development of a pro-metastatic phenotype in TAMs, which specifically in hypoxic areas of the tumor helped tumor cells invade into the vasculature[64]. In rodent models it is however difficult to control tumor hypoxia levels, and 
therefore difficult to isolate the effects of hypoxia from the many coinciding pathological signaling pathways which are active in tumors. We have therefore utilized our hypoxia-model in zebrafish embryos to study the pro-metastatic effects specifically induced by hypoxia[15, 65]. As zebrafish embryos are completely transparent, tumor cell movements can be followed at the single cell level[66] and even in real time. To our knowledge, there are no other models available that allow visualization of the earliest stages of metastasis, in which single tumor cells disseminate to form micro-metastatic lesions in peripheral tissues. Using this unique model, we could identify a hypoxia-induced VEGF-A-VEGFR2 signaling pathway, which was absolutely crucial for allowing entry of tumor cells into the vasculature. Blockade of VEGFR2 signaling either pharmacologically or by RNA-interference mediated knock-down in the zebrafish host, almost completely blocked hypoxia-induced tumor cell dissemination to distal regions in the zebrafish larvae[14]. Reversely, inducing this pathway by overexpressing VEGF-A in otherwise non-metastatic tumor cells led to pronounced distal dissemination even in normoxia[14]. The importance of this pathway in tumor metastasis has been strengthened by findings in mice that tumor-derived VEGF is an important pro-metastatic factor [67] and that tumors exhibiting a higher degree of hypoxia are also more metastatic[44]. Using the zebrafish model, we could for the first time document the importance and inter-relationship between those two pathways during the early metastatic process.

While the zebrafish model is useful for analyzing mechanisms behind early steps of tumor dissemination and metastasis, and especially useful for studying the effects of hypoxia in this process, it is not well suited for studies on tumor growth or late-stage metastasis such as regrowth of secondary lesions in the metastatic niche. The assay we have developed is fast, taking only three days before a robust metastatic readout can be detected, but this is too short a time for regrowth of the metastatic lesions. As such, for thorough analysis of also later stages in the metastatic process other animal models such as 
experimental or spontaneous metastasis models in mice should be used[44, 68]. In such models, tumor cells are either injected intravenously[68] or tumors are first established as spontaneously occurring tumors in transgenic mice [69], orthotropic tumors or as xenografts[70] which will secondarily give rise to tumor cells in the blood or lymph stream. The blood-borne tumor cells will eventually settle in metastatic niches often either in the liver, lung or lymph nodes, where they will grow into macroscopically detectable masses within $2-8$ weeks[44, 68, 70, 71]. The regrowth of metastatic lesions in these models often follow a prototypical path which allows researchers to determine whether this process may be accelerated or inhibited by certain treatments[44]. As many cancer patients already have tumor cells present in circulation upon diagnosis[72], these models may address the clinically relevant question of how to inhibit growth of metastatic lesions once hematological or lymphatic dissemination has already occurred, in order to slow down disease progression.

Hypoxia may not only affect the growth and permeability of the blood vessels, it may also change the tumor cells themselves making them more fit for survival and growth in the harsh tumor microenvironment, as well as for survival in circulation and endow them with the ability to successfully colonize metastatic nieces. Hypoxia leads to a switch in metabolism in which tumor cells primarily utilize glycolysis rather than oxidative metabolism for energy production[73]. This switch seems to persist even after successful recruitment of new blood vessels to the tumor, which return the oxygen concentration back to normal levels (a phenomenon known as the Warburg effect[74]). This long-lasting effect may be a consequence of a hypoxia-induced metaplastic switch leading to tumor cell de-differentiation, as a similar predominantly glycolytic profile is found in non-malignant stem and progenitor cells[75] including mesenchymal cells[76]. Most types of stem- and progenitor cells require hypoxia for maintaining their de-differentiated phenotype long-term[76], but also seem to require contact with endothelial cells - at least in vivo as they are often found in the so-called 
vascular or peri-vascular niche[77]. These seemingly contradictory findings lead to the hypothesis that induction/maintenance of a de-differentiated cell-fate may require hypoxia, but that such a cellular phenotype may persist for some time in normoxia (figure 4). As mentioned above, tumor blood vessels exhibit chaotic circulation, which is frequently changing between non- and re-perfusion. Such chaotic blood flow pattern will lead to sharp hypoxia-reoxygenation cycles which generate very high intracellular ROS in the tumor cells[78]. ROS have been found to bring about many of the same changes in the tumor microenvironment as hypoxia, including tumor cell de-differentiation and metastasis (reviewed in [79]). Thus, hypoxia/ROS-induced vascular malformations and chaotic blood flow may synergize with hypoxia/ROS-induced EMT to promote metastasis. EMT is associated with a gain in motility due to a switch from epithelial-type E-cadherin to mesenchymal-type N-cadherin. Tumor cells, which in their epithelial state are firmly attached within their microenvironment thus, in their mesenchymal state, start to penetrate into the surrounding tissue toward the vasculature. However, should the mesenchymal phenotype be lost upon reoxygenation, the tumor cells would not maintain such an invasive phenotype once they have moved into close proximity of the oxygen-rich perfused vasculature. Thus, the switch has to endure at least for some time following reoxygenation. The hypoxia-induced changes and de-differentiation of tumor cells is probably, however, eventually reversible. As the metastasized tumor cells arrive at the metastatic niche, they extravasate, but then stay in close contact with the endothelium. As such, the oxygen-rich new environment in the micrometastatic lesion may be a factor in driving a mesenchymal-to-epithelial transition (MET)[80] just as it is seen in stem- and progenitor cells, which loose their pluripotency faster when cultured in normoxia[81]. This is important for speeding up cell proliferation (epithelial-like tumor cells proliferate faster than mesenchymal-like tumor cells) and establishing a clinically important metastasis; dedifferentiated mesenchymal-like tumor cells 
are not able to cause the development of macro-metastases[82]. As such, oxygen may regulate both EMT and MET in the primary tumor or in micro-metastases respectively, both processes being involved in development of clinically detectable metastases (Figure 4). As such, the levels of hypoxia/normoxia may drive dynamic, but relatively long-lived changes in tumor cell differentiation/de-differentiation, which are crucial for progression to metastatic disease. Due to the detrimental effects of hypoxia, strategies, which aim at improving tumor oxygenation, through "normalizing" the tumor vasculature and improving perfusion, have been shown to bring about a dramatic reduction in metastasis[83]. Such changes are furthermore coupled to a heightened sensitivity to treatment leading to improved responseand longer survival rates $[84,85]$. These findings have changed the way that researchers think about anti-angiogenic therapy. It should perhaps not be the goal of anti-angiogenic therapy to completely eliminate the tumor vasculature, which would create a situation in which the degenerating vasculature leave holes in the endothelium allowing easier invasion of the vasculature, in which therapeutic drugs would no longer be able to enter into the tumor and in which the tumor cells would experience extreme hypoxia leading to a conversion to a more invasive phenotype. Instead, sub-maximal dosing of anti-angiogenic compounds (which would also give less side-effects, especially after prolonged treatment) could provide a more efficient anti-cancer benefit, especially in combination with cytostatics by simply pruning the pathological vessels, but leaving a "high-quality" vasculature still intact and functional within the tumor. Whether such a schedule would be more or less effective for clinical management of cancer, however, remains untested.

4. Conclusions and further directions 
Circadian and hypoxia signaling are two cornerstones in tumor cell metastasis regulating not only pathological changes in tumor cell metabolism, de-differentiation and acquisition of an invasive phenotype, but also pro-metastatic changes in tumor stroma cells including macrophages and endothelial cells, which synergize to drive systemic spread in cancer[2, 4]. Both of these major pathways can be disrupted on multiple levels in cancer. Genes encoding critical signaling factors such as Clock or Period2 in the circadian clock or VHL in the hypoxia-signaling pathway are frequently mutated in some cancers, but in many cases such genetic aberrations in the tumor cells may not be clear. The tumor vasculature is critically involved in coupling the tumor to endocrine circadian entraining factors needed for the tumor cells to tell the time as they do not sense light themselves. Also, tumor vessels are the conduit for oxygen transport and therefore critically involved in oxygenation. The pathological nature of tumor vessels therefore lead to an inability of the tumor cells to be properly entrained by circulating factors or to receive sufficient amounts of oxygen, even when the tumor is densely vascularized. Therefore, the tumor vasculature can be considered a master regulator of tumor metastasis not only because they provide the passive conduit for systemic dissemination of tumor cells but also because the pathological functions in tumors lead to circadian disruptionand hypoxia-induced de-differentiation coupled to pro-invasive behavior in the tumor cells themselves (Figure 4).

Zebrafish possess great advantages for studies on circadian rhythms and hypoxia in vertebrates[10, 38]. In contrast to mammals, the zebrafish embryo is not dependent on circadian rhythmicity of the mother, but instead is directly entrained by light, which furthermore regulates circadian rhythms in all cells of the transparent embryo. As the embryo is small, passive diffusion of oxygen is considered the main route for oxygenation of the cells, circulation is not needed until much later, when the larvae has grown in size. In addition, recent developments in tumor metastasis models using zebrafish embryos indicate 
that this is an eminent system for studies on the mechanisms governing early stages of tumor metastasis[50], while mouse or other metastasis models are still needed for studies on mechanisms governing later stages during the metastatic cascade.

In the future I predict that zebrafish embryos will gain a central role as tools to delineate important mechanisms behind circadian- and hypoxia-induced early metastasis. It will also be of key importance to discover whether disrupted circulation in tumors is de facto involved in tumor cell de-differentiation via disrupting the circadian rhythm. Also there are still many aspects of circadian biology in cancer of potential significance for metastatic progression, which remain under-investigated. To what extend is the circadian clock in tumor cells disrupted by mutations in clock-factors or their regulators, and how does such mutations contribute to the prognosis? To what extent is the tumor clock de-regulated by systemic circadian disruption such as frequent night-shift work, and how does that impact tumor metastasis? Is disruption of circadian rhythmicity in tumors a general phenomenon and essential for the pathology (i.e. a "Hallmark") of cancer? And can disrupted circadian rhythms in tumors be normalized therapeutically? If so, would that lead to improved prognosis?

The role of tumor hypoxia for promoting metastasis has been the topic of a large body of investigations, however, in most of these studies the researchers have either utilized cellbased in vitro models or rodents models to infer their conclusions. I propose that the zebrafish model is superior to these other models as it provides a system for controlling tumor hypoxia in a living animal without affecting any other aspects of tumor biology. This can also be achieved in vitro, but metastasis is a complicated process that is not accurately recapitulated by solely looking at protein expression and cell motility in a plastic dish. In rodents, the effects of hypoxia cannot be isolated from other potentially pro-metastatic factors such as acidosis, lack of shear-stress stimulation of the endothelium leading to vessel degeneration or 
metabolic stress due to insufficient delivery of nutrients. Thus, the zebrafish model combines the best aspects of in vitro and rodent metastasis models, at least for studying early steps in the metastatic cascade, and will therefore provide an important tool for further research. Using this model, it will be important to delineate whether hypoxia signaling in host cells (ie macrophages or endothelial cells) may contribute to tumor metastasis, or if hypoxia-signaling in the tumor cells themselves is sufficient to drive this process. Also as a diagnostic tool, I predict that this model will gain more attention as it may generate a clinically relevant readout concerning the metastatic behavior of patient-derived primary tumor cells, which is today inferred from pathological examination of the cell shape and sometimes gene expression profile. Finally, I think it is important to establish clinically whether sub-maximal anti-angiogenic treatment in cancer may provide clinical benefit over treatment with the maximally tolerated dose - especially in combination with cytostatics - and whether such benefit could be related to a reduction in tumor hypoxia and metastatic induction of the tumor cells.

\section{Acknowledgements}

I would like to thank Jon Nilsson for providing the basis for the artwork in figure 2. Lasse Jensen is supported by Goesta Fraenkels Stiftelse, Svenska Sällskapet för Medicinsk Forskning, Åke Wibergs Stiftelse, Ollie och Elov Ericssons Stiftelse, Linköpings Universitet and Karolinska Institutet.

\section{References}

[1] S.M. Reppert, D.R. Weaver, Coordination of circadian timing in mammals, Nature, 418 (2002) 935-941. 
[2] L. Mao, R.T. Dauchy, D.E. Blask, L.M. Slakey, S. Xiang, L. Yuan, E.M. Dauchy, B. Shan, G.C. Brainard, J.P. Hanifin, T. Frasch, T.T. Duplessis, S.M. Hill, Circadian gating of epithelial-to-mesenchymal transition in breast cancer cells via melatonin-regulation of GSK3beta, Mol Endocrinol, 26 (2012) 1808-1820.

[3] W.W. Hwang-Verslues, P.H. Chang, Y.M. Jeng, W.H. Kuo, P.H. Chiang, Y.C. Chang, T.H. Hsieh, F.Y. Su, L.C. Lin, S. Abbondante, C.Y. Yang, H.M. Hsu, J.C. Yu, K.J. Chang, J.Y. Shew, E.Y. Lee, W.H. Lee, Loss of corepressor PER2 under hypoxia up-regulates OCT1-mediated EMT gene expression and enhances tumor malignancy, Proceedings of the National Academy of Sciences of the United States of America, 110 (2013) 12331-12336.

[4] B. Keith, M.C. Simon, Hypoxia-inducible factors, stem cells, and cancer, Cell, 129 (2007) 465-472.

[5] K. Eckel-Mahan, P. Sassone-Corsi, Metabolism and the circadian clock converge, Physiological reviews, 93 (2013) 107-135.

[6] N.V. Iyer, L.E. Kotch, F. Agani, S.W. Leung, E. Laughner, R.H. Wenger, M. Gassmann, J.D. Gearhart, A.M. Lawler, A.Y. Yu, G.L. Semenza, Cellular and developmental control of $\mathrm{O} 2$ homeostasis by hypoxia-inducible factor 1 alpha, Genes \& development, 12 (1998) 149-162.

[7] Y. Wang, C. Wan, L. Deng, X. Liu, X. Cao, S.R. Gilbert, M.L. Bouxsein, M.C. Faugere, R.E. Guldberg, L.C. Gerstenfeld, V.H. Haase, R.S. Johnson, E. Schipani, T.L. Clemens, The hypoxia-inducible factor alpha pathway couples angiogenesis to osteogenesis during skeletal development, The Journal of clinical investigation, 117 (2007) 1616-1626.

[8] D.E. Swinson, J.L. Jones, D. Richardson, C. Wykoff, H. Turley, J. Pastorek, N. Taub, A.L. Harris, K.J. O'Byrne, Carbonic anhydrase IX expression, a novel surrogate marker of tumor hypoxia, is associated with a poor prognosis in non-small-cell lung cancer, Journal of clinical oncology : official journal of the American Society of Clinical Oncology, 21 (2003) 473-482.

[9] L.D. Jensen, P. Rouhi, Z. Cao, T. Lanne, E. Wahlberg, Y. Cao, Zebrafish models to study hypoxiainduced pathological angiogenesis in malignant and nonmalignant diseases, Birth defects research. Part C, Embryo today : reviews, 93 (2011) 182-193.

[10] L.D. Jensen, Y. Cao, Clock controls angiogenesis, Cell Cycle, 12 (2013) 405-408.

[11] L. Dahl Ejby Jensen, R. Cao, E.M. Hedlund, I. Soll, J.O. Lundberg, G. Hauptmann, J.F. Steffensen, Y. Cao, Nitric oxide permits hypoxia-induced lymphatic perfusion by controlling arterial-lymphatic conduits in zebrafish and glass catfish, Proceedings of the National Academy of Sciences of the United States of America, 106 (2009) 18408-18413.

[12] C. Gray, I.M. Packham, F. Wurmser, N.C. Eastley, P.G. Hellewell, P.W. Ingham, D.C. Crossman, T.J. Chico, Ischemia is not required for arteriogenesis in zebrafish embryos, Arteriosclerosis, thrombosis, and vascular biology, 27 (2007) 2135-2141.

[13] B.M. Weinstein, D.L. Stemple, W. Driever, M.C. Fishman, Gridlock, a localized heritable vascular patterning defect in the zebrafish, Nature medicine, 1 (1995) 1143-1147.

[14] S.L. Lee, P. Rouhi, L. Dahl Jensen, D. Zhang, H. Ji, G. Hauptmann, P. Ingham, Y. Cao, Hypoxiainduced pathological angiogenesis mediates tumor cell dissemination, invasion, and metastasis in a zebrafish tumor model, Proceedings of the National Academy of Sciences of the United States of America, 106 (2009) 19485-19490.

[15] P. Rouhi, L.D. Jensen, Z. Cao, K. Hosaka, T. Lanne, E. Wahlberg, J.F. Steffensen, Y. Cao, Hypoxiainduced metastasis model in embryonic zebrafish, Nature protocols, 5 (2010) 1911-1918.

[16] U. Schibler, P. Sassone-Corsi, A web of circadian pacemakers, Cell, 111 (2002) 919-922.

[17] M. Akashi, Y. Tsuchiya, T. Yoshino, E. Nishida, Control of intracellular dynamics of mammalian period proteins by casein kinase I epsilon (CKIepsilon) and CKIdelta in cultured cells, Molecular and cellular biology, 22 (2002) 1693-1703.

[18] E.J. Eide, E.L. Vielhaber, W.A. Hinz, D.M. Virshup, The circadian regulatory proteins BMAL1 and cryptochromes are substrates of casein kinase lepsilon, The Journal of biological chemistry, 277 (2002) 17248-17254. 
[19] N.C. Gossan, F. Zhang, B. Guo, D. Jin, H. Yoshitane, A. Yao, N. Glossop, Y.Q. Zhang, Y. Fukada, Q.J. Meng, The E3 ubiquitin ligase UBE3A is an integral component of the molecular circadian clock through regulating the BMAL1 transcription factor, Nucleic acids research, 42 (2014) 5765-5775. [20] S.M. Siepka, S.H. Yoo, J. Park, W. Song, V. Kumar, Y. Hu, C. Lee, J.S. Takahashi, Circadian mutant Overtime reveals F-box protein FBXL3 regulation of cryptochrome and period gene expression, Cell, 129 (2007) 1011-1023.

[21] E. Maury, K.M. Ramsey, J. Bass, Circadian rhythms and metabolic syndrome: from experimental genetics to human disease, Circulation research, 106 (2010) 447-462.

[22] N. Wang, G. Yang, Z. Jia, H. Zhang, T. Aoyagi, S. Soodvilai, J.D. Symons, J.B. Schnermann, F.J. Gonzalez, S.E. Litwin, T. Yang, Vascular PPARgamma controls circadian variation in blood pressure and heart rate through Bmal1, Cell metabolism, 8 (2008) 482-491.

[23] S. Shimba, N. Ishii, Y. Ohta, T. Ohno, Y. Watabe, M. Hayashi, T. Wada, T. Aoyagi, M. Tezuka, Brain and muscle Arnt-like protein-1 (BMAL1), a component of the molecular clock, regulates adipogenesis, Proceedings of the National Academy of Sciences of the United States of America, 102 (2005) 12071-12076.

[24] J.E. Gibbs, J. Blaikley, S. Beesley, L. Matthews, K.D. Simpson, S.H. Boyce, S.N. Farrow, K.J. Else, D. Singh, D.W. Ray, A.S. Loudon, The nuclear receptor REV-ERBalpha mediates circadian regulation of innate immunity through selective regulation of inflammatory cytokines, Proceedings of the National Academy of Sciences of the United States of America, 109 (2012) 582-587.

[25] E.S. Musiek, M.M. Lim, G. Yang, A.Q. Bauer, L. Qi, Y. Lee, J.H. Roh, X. Ortiz-Gonzalez, J.T. Dearborn, J.P. Culver, E.D. Herzog, J.B. Hogenesch, D.F. Wozniak, K. Dikranian, B.I. Giasson, D.R. Weaver, D.M. Holtzman, G.A. Fitzgerald, Circadian clock proteins regulate neuronal redox homeostasis and neurodegeneration, The Journal of clinical investigation, 123 (2013) 5389-5400.

[26] L. Fu, C.C. Lee, The circadian clock: pacemaker and tumour suppressor, Nature reviews. Cancer, 3 (2003) 350-361.

[27] G. Oprea-llies, E. Haus, L. Sackett-Lundeen, Y. Liu, L. McLendon, R. Busch, A. Adams, C. Cohen, Expression of melatonin receptors in triple negative breast cancer (TNBC) in African American and Caucasian women: relation to survival, Breast cancer research and treatment, 137 (2013) 677-687. [28] L.D. Jensen, Z. Cao, M. Nakamura, Y. Yang, L. Brautigam, P. Andersson, Y. Zhang, E. Wahlberg, T. Lanne, K. Hosaka, Y. Cao, Opposing effects of circadian clock genes bmal1 and period 2 in regulation of VEGF-dependent angiogenesis in developing zebrafish, Cell reports, 2 (2012) 231-241.

[29] S. Rao, C. Chun, J. Fan, J.M. Kofron, M.B. Yang, R.S. Hegde, N. Ferrara, D.R. Copenhagen, R.A. Lang, A direct and melanopsin-dependent fetal light response regulates mouse eye development, Nature, 494 (2013) 243-246.

[30] S. Koyanagi, Y. Kuramoto, H. Nakagawa, H. Aramaki, S. Ohdo, S. Soeda, H. Shimeno, A molecular mechanism regulating circadian expression of vascular endothelial growth factor in tumor cells, Cancer research, 63 (2003) 7277-7283.

[31] L. Fu, H. Pelicano, J. Liu, P. Huang, C. Lee, The circadian gene Period2 plays an important role in tumor suppression and DNA damage response in vivo, Cell, 111 (2002) 41-50.

[32] K. Miyazaki, M. Wakabayashi, Y. Hara, N. Ishida, Tumor growth suppression in vivo by overexpression of the circadian component, PER2, Genes to cells : devoted to molecular \& cellular mechanisms, 15 (2010) 351-358.

[33] E. Frigato, L. Lunghi, M.E. Ferretti, C. Biondi, C. Bertolucci, Evidence for circadian rhythms in human trophoblast cell line that persist in hypoxia, Biochemical and biophysical research communications, 378 (2009) 108-111.

[34] N. Takeda, K. Maemura, S. Horie, K. Oishi, Y. Imai, T. Harada, T. Saito, T. Shiga, E. Amiya, I. Manabe, N. Ishida, R. Nagai, Thrombomodulin is a clock-controlled gene in vascular endothelial cells, The Journal of biological chemistry, 282 (2007) 32561-32567.

[35] Y. Wang, L. Hua, C. Lu, Z. Chen, Expression of circadian clock gene human Period2 (hPer2) in human colorectal carcinoma, World journal of surgical oncology, 9 (2011) 166. 
[36] L. Wang, B. Chen, Y. Wang, N. Sun, C. Lu, R. Qian, L. Hua, hClock gene expression in human colorectal carcinoma, Molecular medicine reports, 8 (2013) 1017-1022.

[37] C.Y. Wang, M.S. Wen, H.W. Wang, I.C. Hsieh, Y. Li, P.Y. Liu, F.C. Lin, J.K. Liao, Increased vascular senescence and impaired endothelial progenitor cell function mediated by mutation of circadian gene Per2, Circulation, 118 (2008) 2166-2173.

[38] C.B. Anea, B. Cheng, S. Sharma, S. Kumar, R.W. Caldwell, L. Yao, M.I. Ali, A.M. Merloiu, D.W. Stepp, S.M. Black, D.J. Fulton, R.D. Rudic, Increased superoxide and endothelial NO synthase uncoupling in blood vessels of Bmal1-knockout mice, Circulation research, 111 (2012) 1157-1165. [39] R.S. Edgar, E.W. Green, Y. Zhao, G. van Ooijen, M. Olmedo, X. Qin, Y. Xu, M. Pan, U.K. Valekunja, K.A. Feeney, E.S. Maywood, M.H. Hastings, N.S. Baliga, M. Merrow, A.J. Millar, C.H. Johnson, C.P. Kyriacou, J.S. O'Neill, A.B. Reddy, Peroxiredoxins are conserved markers of circadian rhythms, Nature, 485 (2012) 459-464.

[40] Y. Xue, P. Religa, R. Cao, A.J. Hansen, F. Lucchini, B. Jones, Y. Wu, Z. Zhu, B. Pytowski, Y. Liang, W. Zhong, P. Vezzoni, B. Rozell, Y. Cao, Anti-VEGF agents confer survival advantages to tumorbearing mice by improving cancer-associated systemic syndrome, Proceedings of the National Academy of Sciences of the United States of America, 105 (2008) 18513-18518.

[41] L.J. Nissen, R. Cao, E.M. Hedlund, Z. Wang, X. Zhao, D. Wetterskog, K. Funa, E. Brakenhielm, Y. Cao, Angiogenic factors FGF2 and PDGF-BB synergistically promote murine tumor neovascularization and metastasis, The Journal of clinical investigation, 117 (2007) 2766-2777.

[42] H.F. Dvorak, Tumors: wounds that do not heal. Similarities between tumor stroma generation and wound healing, The New England journal of medicine, 315 (1986) 1650-1659.

[43] R.K. Jain, Normalization of tumor vasculature: an emerging concept in antiangiogenic therapy, Science, 307 (2005) 58-62.

[44] K. Hosaka, Y. Yang, T. Seki, M. Nakamura, P. Andersson, P. Rouhi, X. Yang, L. Jensen, S. Lim, N. Feng, Y. Xue, X. Li, O. Larsson, T. Ohhashi, Y. Cao, Tumour PDGF-BB expression levels determine dual effects of anti-PDGF drugs on vascular remodelling and metastasis, Nature communications, 4 (2013) 2129.

[45] A.J. Maniotis, R. Folberg, A. Hess, E.A. Seftor, L.M. Gardner, J. Pe'er, J.M. Trent, P.S. Meltzer, M.J. Hendrix, Vascular channel formation by human melanoma cells in vivo and in vitro: vasculogenic mimicry, The American journal of pathology, 155 (1999) 739-752.

[46] G.M. Tozer, C. Kanthou, B.C. Baguley, Disrupting tumour blood vessels, Nature reviews. Cancer, 5 (2005) 423-435.

[47] C.B. Anea, M.X. Zhang, F. Chen, M.I. Ali, C.M.M. Hart, D.W. Stepp, Y.O. Kovalenkov, A.M. Merloiu, P. Pati, D. Fulton, R.D. Rudic, Circadian Clock Control of Nox4 and Reactive Oxygen Species in the Vasculature, PloS one, 8 (2013).

[48] Y. Okuno, A. Nakamura-Ishizu, K. Otsu, T. Suda, Y. Kubota, Pathological neoangiogenesis depends on oxidative stress regulation by ATM, Nature medicine, 18 (2012) 1208-+.

[49] Q. Ke, M. Costa, Hypoxia-inducible factor-1 (HIF-1), Molecular pharmacology, 70 (2006) 14691480.

[50] P. Rouhi, S.L. Lee, Z. Cao, E.M. Hedlund, L.D. Jensen, Y. Cao, Pathological angiogenesis facilitates tumor cell dissemination and metastasis, Cell Cycle, 9 (2010) 913-917.

[51] L.D. Jensen, R. Cao, Y. Cao, In vivo angiogenesis and lymphangiogenesis models, Current molecular medicine, 9 (2009) 982-991.

[52] G.L. Semenza, Regulation of mammalian 02 homeostasis by hypoxia-inducible factor 1 , Annual review of cell and developmental biology, 15 (1999) 551-578.

[53] W.R. Wilson, M.P. Hay, Targeting hypoxia in cancer therapy, Nature reviews. Cancer, 11 (2011) 393-410.

[54] P.A. Padilla, M.B. Roth, Oxygen deprivation causes suspended animation in the zebrafish embryo, Proceedings of the National Academy of Sciences of the United States of America, 98 (2001) 7331-7335. 
[55] Z. Cao, L.D. Jensen, P. Rouhi, K. Hosaka, T. Lanne, J.F. Steffensen, E. Wahlberg, Y. Cao, Hypoxiainduced retinopathy model in adult zebrafish, Nature protocols, 5 (2010) 1903-1910.

[56] I.J. Marques, J.T. Leito, H.P. Spaink, J. Testerink, R.T. Jaspers, F. Witte, S. van den Berg, C.P. Bagowski, Transcriptome analysis of the response to chronic constant hypoxia in zebrafish hearts, Journal of comparative physiology. B, Biochemical, systemic, and environmental physiology, 178 (2008) 77-92.

[57] E. Jacob, M. Drexel, T. Schwerte, B. Pelster, Influence of hypoxia and of hypoxemia on the development of cardiac activity in zebrafish larvae, American journal of physiology. Regulatory, integrative and comparative physiology, 283 (2002) R911-917.

[58] E. van Rooijen, E.E. Voest, I. Logister, J. Bussmann, J. Korving, F.J. van Eeden, R.H. Giles, S. Schulte-Merker, von Hippel-Lindau tumor suppressor mutants faithfully model pathological hypoxiadriven angiogenesis and vascular retinopathies in zebrafish, Disease models \& mechanisms, 3 (2010) 343-353.

[59] E. van Rooijen, E.E. Voest, I. Logister, J. Korving, T. Schwerte, S. Schulte-Merker, R.H. Giles, F.J. van Eeden, Zebrafish mutants in the von Hippel-Lindau tumor suppressor display a hypoxic response and recapitulate key aspects of Chuvash polycythemia, Blood, 113 (2009) 6449-6460.

[60] C. Jopling, G. Sune, A. Faucherre, C. Fabregat, J.C. Izpisua Belmonte, Hypoxia induces myocardial regeneration in zebrafish, Circulation, 126 (2012) 3017-3027.

[61] J. Folkman, Tumor angiogenesis: therapeutic implications, The New England journal of medicine, 285 (1971) 1182-1186.

[62] G. Bergers, D. Hanahan, Modes of resistance to anti-angiogenic therapy, Nature reviews. Cancer, 8 (2008) 592-603.

[63] J.W. Pollard, Tumour-educated macrophages promote tumour progression and metastasis, Nature reviews. Cancer, 4 (2004) 71-78.

[64] A. Casazza, D. Laoui, M. Wenes, S. Rizzolio, N. Bassani, M. Mambretti, S. Deschoemaeker, J.A. Van Ginderachter, L. Tamagnone, M. Mazzone, Impeding macrophage entry into hypoxic tumor areas by Sema3A/Nrp1 signaling blockade inhibits angiogenesis and restores antitumor immunity, Cancer cell, 24 (2013) 695-709.

[65] L. Gomez-Maldonado, M. Tiana, O. Roche, A. Prado-Cabrero, L. Jensen, A. Fernandez-Barral, I. Guijarro-Munoz, E. Favaro, G. Moreno-Bueno, L. Sanz, J. Aragones, A. Harris, O. Volpert, B. Jimenez, L. Del Peso, EFNA3 long noncoding RNAs induced by hypoxia promote metastatic dissemination, Oncogene, 0 (2014).

[66] A. Fernandez-Barral, J.L. Orgaz, P. Baquero, Z. Ali, A. Moreno, M. Tiana, V. Gomez, E. RiveiroFalkenbach, C. Canadas, S. Zazo, C. Bertolotto, I. Davidson, J.L. Rodriguez-Peralto, I. Palmero, F. Rojo, L.D. Jensen, L. Del Peso, B. Jimenez, Regulatory and functional connection of microphthalmiaassociated transcription factor and anti-metastatic pigment epithelium derived factor in melanoma, Neoplasia, 16 (2014) 529-542.

[67] M.A. Bjorndahl, R. Cao, J.B. Burton, E. Brakenhielm, P. Religa, D. Galter, L. Wu, Y. Cao, Vascular endothelial growth factor-a promotes peritumoral lymphangiogenesis and lymphatic metastasis, Cancer research, 65 (2005) 9261-9268.

[68] J.M. Ebos, C.R. Lee, W. Cruz-Munoz, G.A. Bjarnason, J.G. Christensen, R.S. Kerbel, Accelerated metastasis after short-term treatment with a potent inhibitor of tumor angiogenesis, Cancer cell, 15 (2009) 232-239.

[69] M. Paez-Ribes, E. Allen, J. Hudock, T. Takeda, H. Okuyama, F. Vinals, M. Inoue, G. Bergers, D. Hanahan, O. Casanovas, Antiangiogenic therapy elicits malignant progression of tumors to increased local invasion and distant metastasis, Cancer cell, 15 (2009) 220-231.

[70] H. Ji, R. Cao, Y. Yang, Y. Zhang, H. Iwamoto, S. Lim, M. Nakamura, P. Andersson, J. Wang, Y. Sun, S. Dissing, X. He, X. Yang, Y. Cao, TNFR1 mediates TNF-alpha-induced tumour lymphangiogenesis and metastasis by modulating VEGF-C-VEGFR3 signalling, Nature communications, 5 (2014) 4944. 
[71] R. Cao, M.A. Bjorndahl, P. Religa, S. Clasper, S. Garvin, D. Galter, B. Meister, F. Ikomi, K. Tritsaris, S. Dissing, T. Ohhashi, D.G. Jackson, Y. Cao, PDGF-BB induces intratumoral lymphangiogenesis and promotes lymphatic metastasis, Cancer cell, 6 (2004) 333-345.

[72] S.P. Leong, W.W. Tseng, Micrometastatic cancer cells in lymph nodes, bone marrow, and blood: Clinical significance and biologic implications, CA: a cancer journal for clinicians, 64 (2014) 195-206.

[73] G.L. Semenza, HIF-1 mediates metabolic responses to intratumoral hypoxia and oncogenic mutations, The Journal of clinical investigation, 123 (2013) 3664-3671.

[74] O. Warburg, On the origin of cancer cells, Science, 123 (1956) 309-314.

[75] T. Simsek, F. Kocabas, J. Zheng, R.J. Deberardinis, A.I. Mahmoud, E.N. Olson, J.W. Schneider, C.C. Zhang, H.A. Sadek, The distinct metabolic profile of hematopoietic stem cells reflects their location in a hypoxic niche, Cell stem cell, 7 (2010) 380-390.

[76] K. Ito, T. Suda, Metabolic requirements for the maintenance of self-renewing stem cells, Nature reviews. Molecular cell biology, 15 (2014) 243-256.

[77] S. Shi, S. Gronthos, Perivascular niche of postnatal mesenchymal stem cells in human bone marrow and dental pulp, Journal of bone and mineral research : the official journal of the American Society for Bone and Mineral Research, 18 (2003) 696-704.

[78] M.W. Dewhirst, Y. Cao, B. Moeller, Cycling hypoxia and free radicals regulate angiogenesis and radiotherapy response, Nature reviews. Cancer, 8 (2008) 425-437.

[79] W.S. Wu, The signaling mechanism of ROS in tumor progression, Cancer metastasis reviews, 25 (2006) 695-705.

[80] I. Moen, A.M. Oyan, K.H. Kalland, K.J. Tronstad, L.A. Akslen, M. Chekenya, P.O. Sakariassen, R.K. Reed, L.E. Stuhr, Hyperoxic treatment induces mesenchymal-to-epithelial transition in a rat adenocarcinoma model, PloS one, 4 (2009) e6381.

[81] M.G. Cipolleschi, P. Dello Sbarba, M. Olivotto, The role of hypoxia in the maintenance of hematopoietic stem cells, Blood, 82 (1993) 2031-2037.

[82] D. Yao, C. Dai, S. Peng, Mechanism of the mesenchymal-epithelial transition and its relationship with metastatic tumor formation, Molecular cancer research : MCR, 9 (2011) 1608-1620.

[83] M. Mazzone, D. Dettori, R. Leite de Oliveira, S. Loges, T. Schmidt, B. Jonckx, Y.M. Tian, A.A. Lanahan, P. Pollard, C. Ruiz de Almodovar, F. De Smet, S. Vinckier, J. Aragones, K. Debackere, A. Luttun, S. Wyns, B. Jordan, A. Pisacane, B. Gallez, M.G. Lampugnani, E. Dejana, M. Simons, P. Ratcliffe, P. Maxwell, P. Carmeliet, Heterozygous deficiency of PHD2 restores tumor oxygenation and inhibits metastasis via endothelial normalization, Cell, 136 (2009) 839-851.

[84] E.M. Hedlund, X. Yang, Y. Zhang, Y. Yang, M. Shibuya, W. Zhong, B. Sun, Y. Liu, K. Hosaka, Y. Cao, Tumor cell-derived placental growth factor sensitizes antiangiogenic and antitumor effects of antiVEGF drugs, Proceedings of the National Academy of Sciences of the United States of America, 110 (2013) 654-659.

[85] D. Zhang, E.M. Hedlund, S. Lim, F. Chen, Y. Zhang, B. Sun, Y. Cao, Antiangiogenic agents significantly improve survival in tumor-bearing mice by increasing tolerance to chemotherapyinduced toxicity, Proceedings of the National Academy of Sciences of the United States of America, 108 (2011) 4117-4122.

Figure Legends

Figure 1. Organization of the cellular circadian clock 
Circadian input signals drive RORalpha-mediated production of Bmal1 (purple beans), which is inhibited by Rev-Erbalpha. Bmal1 heterodimerize with Clock or Npas2 (green beans), bind to E-boxes in the promoters of clock-controlled genes (CCGs) and together with co-activators (turquoise shapes) drive transcription. Periods (dark blue shapes) and chryptochromes (yellow shapes) produced in this way feedback onto this regulation and inhibit Bmal1Clock/Npas2-mediated gene transcription. The circadian transcriptional regulators are removed from the cell by ubuiquitin-ligase-mediated targeting for proteasomal degradation.

Figure 2. Regulation of hypoxia signaling

Under normoxia, HIF1alpha proteins (purple beans) are hydroxylated by PHDs or FIH, which in the former case target HIF1alpha for ubuiquitinylation (yellow circles) by VHL and degradation, or in the latter case inhibit the binding of essential transcriptional co-activators. Under hypoxia, HIF1alpha will remain non-hydroxylated and thus stabilized. Under such conditions, HIF1alpha enter the nucleus, heterodimerize with HIF1beta (green beans), bind to hypoxia-responsive elements in the promoters of hypoxia-induced genes and after recruitment of co-factors (turquoise shapes) drive transcription of target genes such as VEGF.

Figure 3. The hypoxia setup

An aquarium is equipped with a magnet-mixer (white bar), an oxygen electrode (dark gray bar) and an air-stone (black circle). Nitrogen perfusion is controlled by a valve connected to a control-unit, which turn on the valve when the oxygen electrode measures dissolved oxygen levels higher than a set value of for example $10 \%$ relative air-saturation. 
Figure 4. Illustration of the proposed mechanism behind tumor dedifferentiation and metastasis.

Epithelial-like tumor cells (green shapes) reside in well-perfused tumor regions in which oxygen and endocrine circadian mediators keep the cells in a differentiated state. In a different region in the tumor, non-perfused vessels will lead to hypoxia and blunted circadian rhythms in endocrine mediators, which will aid in tumor cell dedifferentiation and development of mesenchymal-like tumor cells (blue shapes). These mesenchymal-like tumor cells are more motile, and invade the vasculature leading to systemic dissemination. At the metastatic niche, delivery of oxygen and circadian rhythms of entraining signals is unperturbed forcing the dedifferentiated, mesenchymal-like tumor cells to re-differentiate into epithelial-like cells, which proliferate and give rise to macrometastatic lesions. 
Figure 1

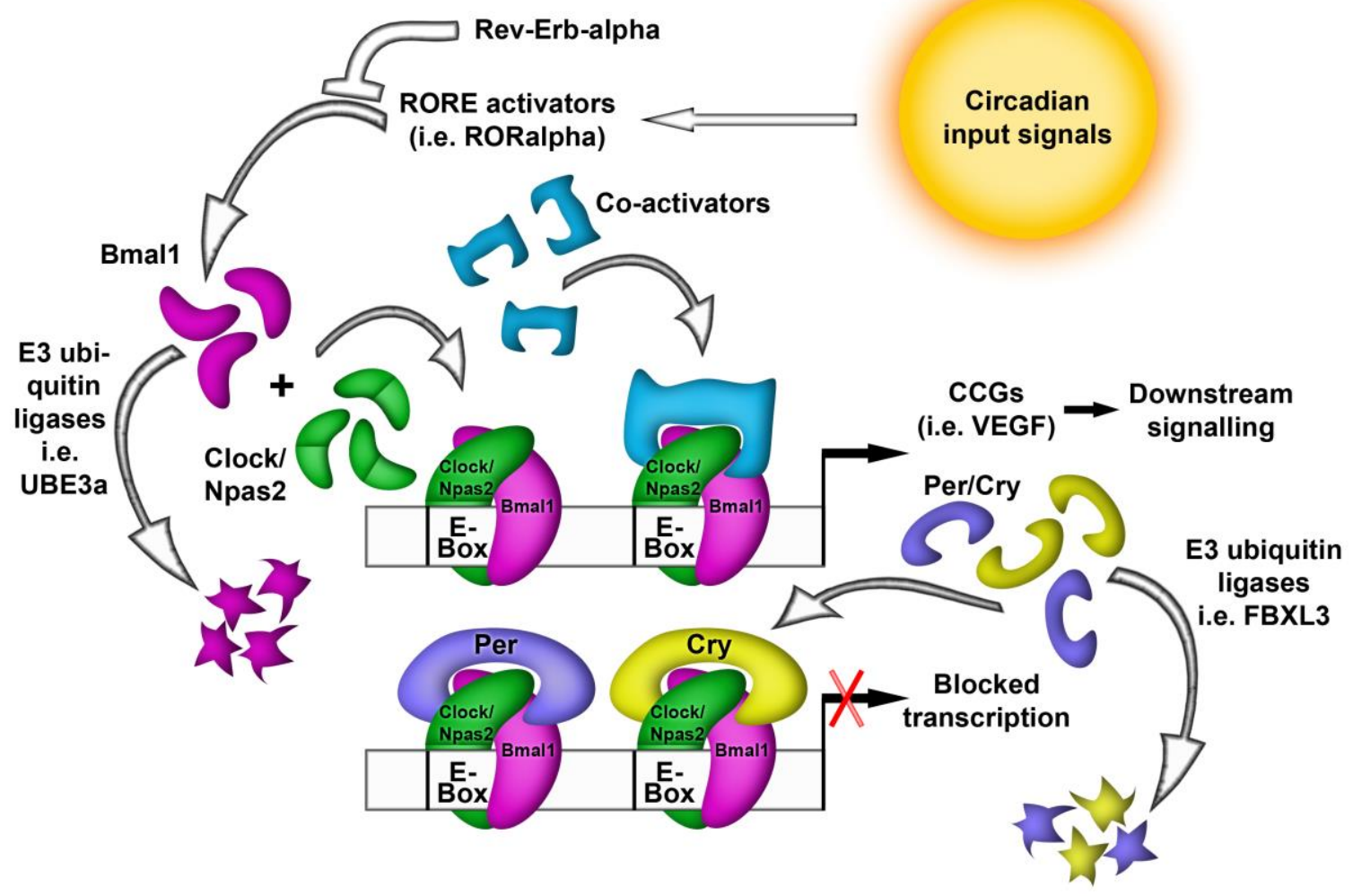


Figure 2

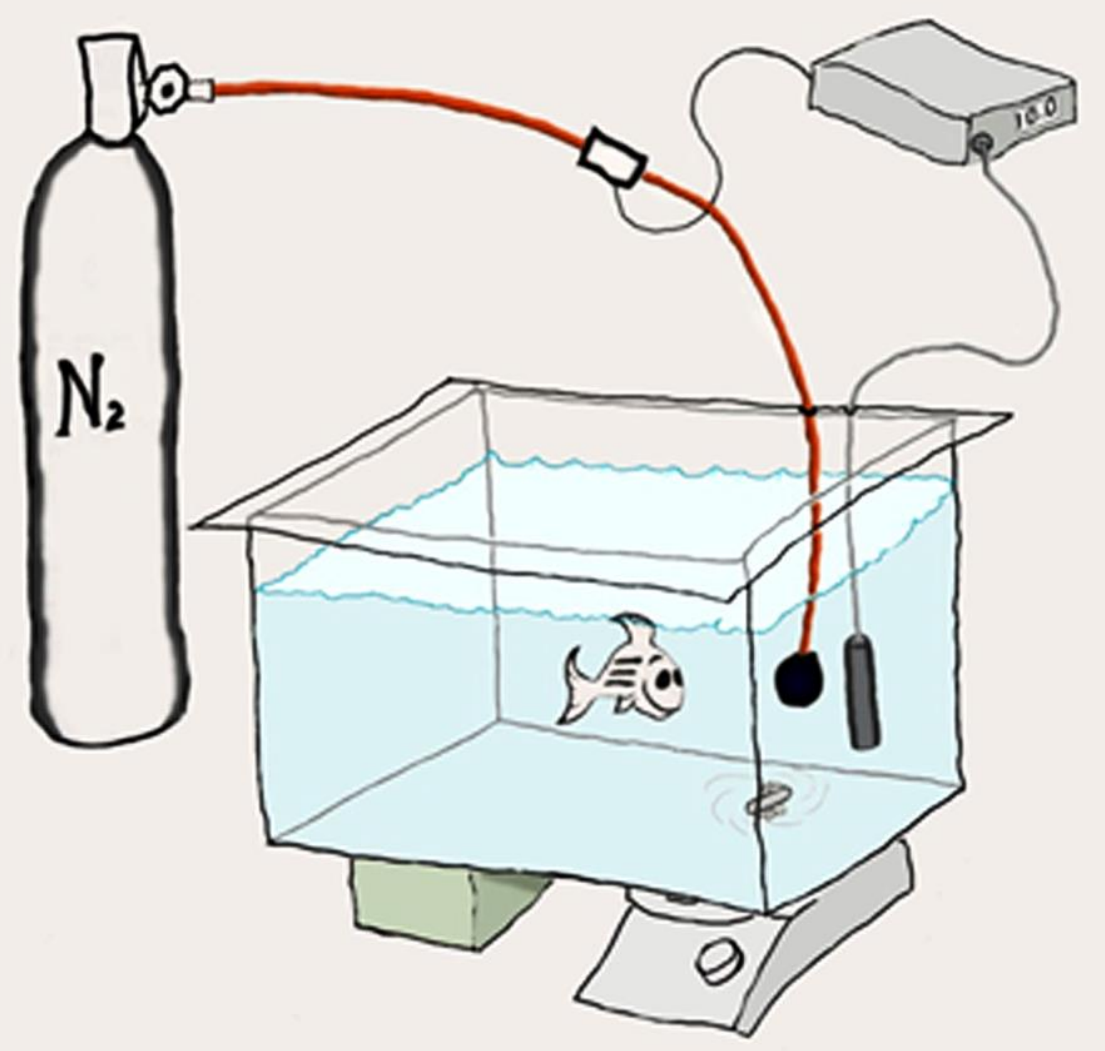


Figure 3

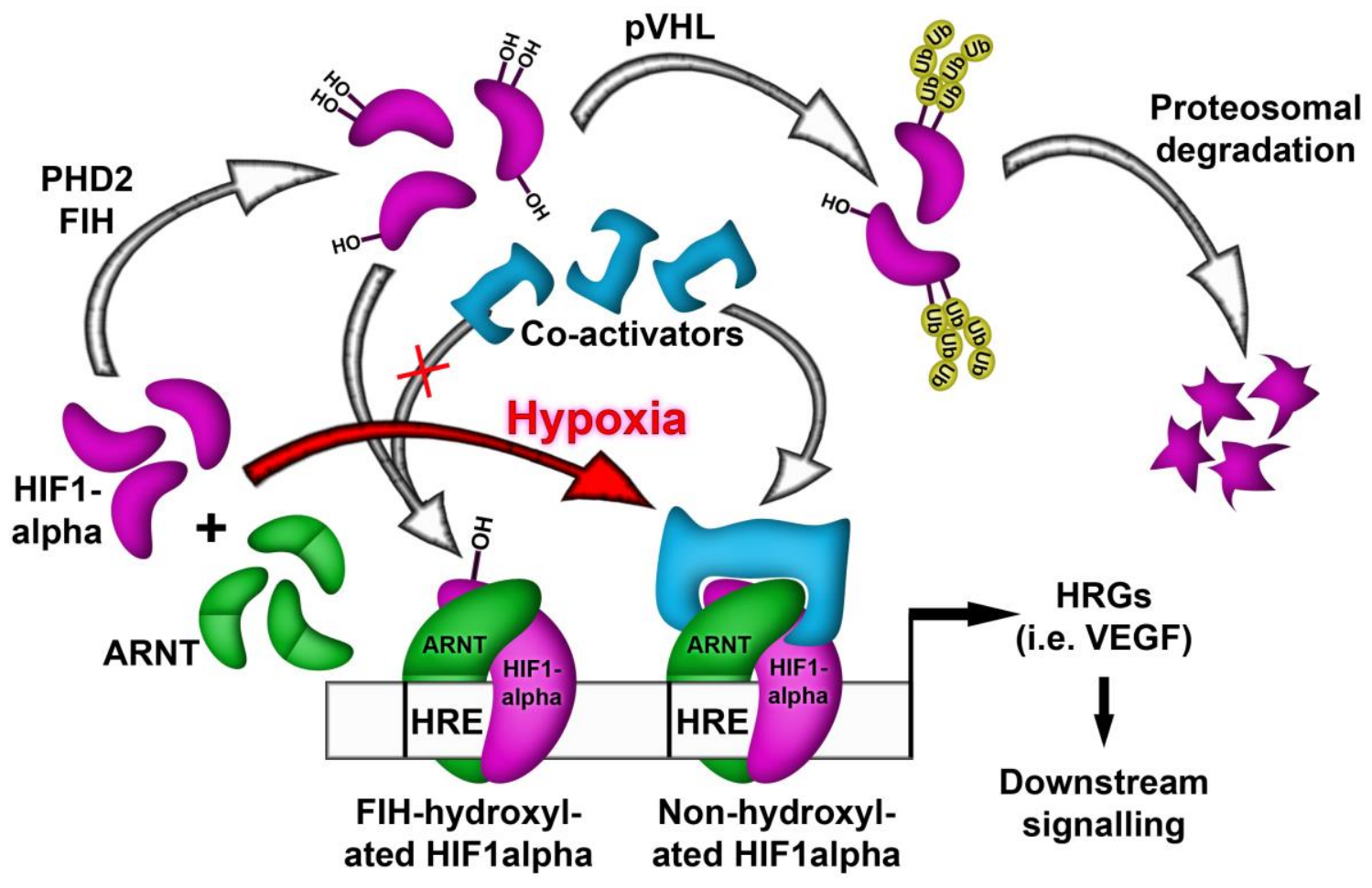


Figure 4
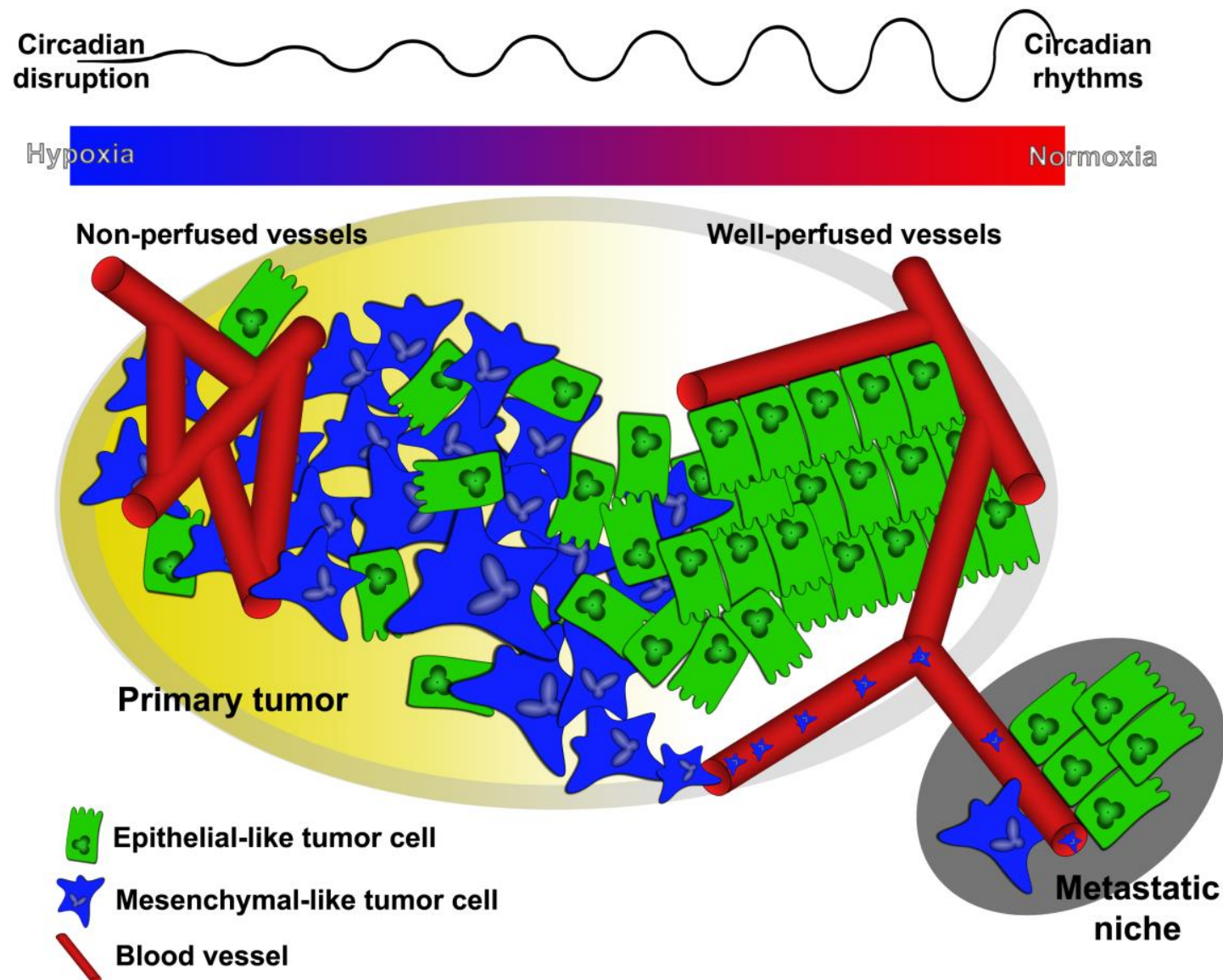